\title{
Pulsed-Laser-Driven TEM for Studying Temporal Aspects of Beam Damage
}

\section{David Flannigan and Elisah VandenBussche}

\section{University of Minnesota, Minneapolis, Minnesota, United States}

Possible mitigation of damage using pulsed electron beams in the TEM has been speculated upon for decades [1]. This stems from hypotheses formulated from time- and energy-dependent processes arising from specimen excitation by the incident electrons. The myriad excitations that can occur are variably operable over a large range of timescales, from femtoseconds to microseconds and longer [2]. Further, the majority of the processes are detrimentally exacerbated (from the perspective of damage) in an exponential manner with subsequent additional energy deposition [3], provided such inputs occur within the spatial region of initial excitation. Such examples include Arrhenius-type reaction rates and diffusion rates of reactive species. Additionally, local excitation of lattice phonons may produce the equivalent of significant local thermal energies that can further drive localized rate increases, despite the equilibrated global thermal-energy bath remaining quite low. Accordingly, more specific hypotheses for mitigating damage using pulsed beams focus on parameters such as the time elapsed between sequential electron arrival at the specimen and the spatiotemporal electron number density.

Here, recent advances in using pulsed beams comrpised of picosecond- to femtosecond-duration electron packets specifically for mitigating damage will be discussed. Though approaches to producing pulsed beams in the TEM have also been explored for decades (see, for example, [4]), more recent advances based upon femtosecond pulsed lasers and high-frequency, RF-modulated chopped beams have introduced high levels of control over elapsed time and electron-packet number density [5-7]. As a result, reports are now beginning to emerge that indeed provide evidence of specimen damage mitigation using temporally-shaped and precisely-modulated pulsed electron beams in the TEM. Initial reports have cited (at times in passing) the observation of inordinate exposure times of beam-sensitive specimens to pulsed beams with little to no reduction in Bragg-spot intensities [8,9]. A more recent study using an RFmodulated beam operated at $\mathrm{GHz}$ repetition rates $(\sim 160 \mathrm{ps}$ between electron packets) reported a significant increase in critical dose and avoidance of a commonly-occurring beam-induced phase transformation in $\mathrm{MgCl}_{2}$ [10]. While intriguing, direct comparisons with conventional (random) electron emission were lacking, wherein pulsed and conventional beams operated at the same dose rate and with accumulation to the same total dose were used to study damage. Such experiments were recently performed on a model beam-sensitive material (hexatriacontane, $\mathrm{C}_{36} \mathrm{H}_{74}$ ), and indeed a significant reduction in damage was observed when using the pulsed beam [11]. Here, in addition to briefly reviewing details of this latter study, a host of experimental pulsed-TEM parameters accessible with interfaced femtosecond pulsed lasers will be described. Analogous to time-domain studies of structural and electronic dynamics with ultrafast electron microscopy [12], the importance of balancing repetition rate, acquisition time, and resolution will be discussed. The apparent implications of these constraints with respect to extending such efforts to high-resolution imaging, electron diffraction, and spectroscopic measurements will also be discussed, especially with respect to varied material types and the host of damage mechanisms that can be activated during inelastic collisions with incident electrons. The goal here is to provide a brief review of the growing efforts in this area while outlining potential opportunities and gaps in current understanding [13].

References

[1] R. A. Bonham and R. E. Kennerly, Ann. N. Y. Acad. Sci. 306 (1978), 85-94.

[2] D. T. Grubb, J. Mater. Sci. 9 (1974), 1715-1736. 
[3] R. F. Egerton, Micron 119 (2019), 72-87.

[4] O. Bostanjoglo and Th. Rosin, Opt. Acta 24 (1977), 657-664.

[5] V. A. Lobastov, R. Srinivasan, and A. H. Zewail, Proc. Natl. Acad. Sci. U.S.A. 102 (2005), 70697073.

[6] J. Qiu, et al., Ultramicroscopy 161 (2016), 130-136.

[7] W. Verhoeven, et al., Ultramicroscopy 188 (2018), 85-89.

[8] D. J. Flannigan, V. A. Lobastov, and A. H. Zewail, Angew. Chem. Int. Ed. 46 (2007), 9206-9210.

[9] O.-H. Kwon, V. Ortalan, and A. H. Zewail, Proc. Natl. Acad. Sci. U.S.A. 108 (2011), 6026-6031.

[10] C. Kisielowski, et al., Adv. Funct. Mater. 29 (2019), 1807818.

[11] E. J. VandenBussche and D. J. Flannigan, Nano Lett. 19 (2019), 6687-6694.

[12] D. A. Plemmons, P. K. Suri, and D. J. Flannigan, Chem. Mater. 27 (2015), 3178-3192.

[13] This material is based upon work supported by the U.S. Department of Energy, Office of Science, Office of Basic Energy Sciences under Award No. DE-SC0018204. This material is based upon work partially supported by the National Science Foundation Graduate Research Fellowship Program under Grant DGE-1348264. Acknowledgment is made to the Donors of the American Chemical Society Petroleum Research Fund for partial support of this research. 